\title{
The Bright and the Slow - GRBs 100724B and 160509A with high-energy cutoffs at $<100 \mathrm{MeV}$
}

\section{G. Vianello*}

HEPL, Stanford University, United States

E-mail: giacomovestanford.edu

\section{R. Gill}

Department of Natural Sciences, The Open University of Israel, Israel Physics Department, Ben-Gurion University, Israel

\section{J. Granot}

Department of Natural Sciences, The Open University of Israel, Israel

\section{N. Omodei}

HEPL, Stanford University, United States

\section{J. Cohen-Tanugi}

Université de Montpellier, CNRS/IN2P3, Montpellier, France

\section{F. Longo}

INFN Trieste, Italy

Universit'a di Trieste, Italy

\begin{abstract}
We analyze the prompt emission of two of the brightest Gamma-Ray Bursts (GRBs) observed by Fermi at MeV energies but surprisingly faint at $>100 \mathrm{MeV}$ energies. Time-resolved spectroscopy reveals a sharp high-energy cutoff. We first characterize phenomenologically the cutoff and its time evolution. We then fit the data to two models where the high-energy cutoff arises from intrinsic opacity to pair production within the source. Alternative explanations for the cutoff, such as an intrinsic cutoff in the emitting electron energy distribution, appear to be less natural. Both models provide a good fit to the data with very reasonable physical parameters, providing a direct estimate of bulk Lorentz factors on the lower end of what is generally observed in Fermi GRBs. Particularly low $E_{c}$ values may prevent detection by Fermi-LAT, thus introducing a bias in the Fermi-LAT GRB sample against GRBs with low Lorentz factors or variability times.
\end{abstract}

7th Fermi Symposium 2017

15-20 October 2017

Garmisch-Partenkirchen, Germany

\footnotetext{
*Speaker.
} 


\section{Introduction}

Long Gamma-Ray Bursts are thought to be produced during the collapse of a very massive star, and specifically from within the relativistic jet that is produced during or immediately after the collapse. However, the mechanism capable of accelerating the jet as well as the process responsible for extracting energy from the jet and radiating it in $\gamma$-rays are still debated [1]. In the context of fireball models, where the jet is barionic and moving with a large Lorentz factor, the highly-variable prompt signal is often attributed to synchrotron emission produced in so-called internal shocks. These ultra-relativistic shocks are produced by the collision within the jet occurring when a faster part of the ejecta catches up with a slower part of the jet. This naturally generates non-thermal spectra similar to what is observed in GRBs. However, this scenario presents two main problems: i) the efficiency of internal shocks in extracting energy from the jet is limited and it does not seem to be enough to explain the flux observed in some GRBs, unless the difference in Lorentz factor between different parts of the jet is large [2]; ii) the non-thermal spectrum of some GRBs does not resemble the spectrum expected from synchrotron emission [3, 4, 5]. In order to overcome these problems, a new class of fireball models has emerged. We will call them for simplicity "photospheric models". In these models, the non-thermal spectrum observed in GRBs is the result of the reprocessing within the jet of an initial thermal or quasi-thermal spectrum coming from the photosphere. There are many photospheric models, differing for the mechanisms and processes responsible for the reprocessing of the thermal spectrum and consequently for the required characteristics of the jet (baryon poor or baryon dominated, magnetized or non-magnetized, and so on). Many of these models predict a spectral cutoff between few $\mathrm{MeV}$ and few $\mathrm{GeV}[6,7,8]$.

\section{GRB 100724B and GRB 160509A}

In this contribution we analyze the prompt emission of two bright GRBs detected by the Fermi Gamma-ray Space Telescope, namely GRB 100724B and GRB 160509A. Details about the analysis and the results can be found in [9]. In this contribution we summarize the main results.

GRB 100724B and GRB 160509A are unique in that they present a very bright low-energy emission, as observed by the Gamma-ray Burst Monitor (GBM) onboard Fermi, but a faint highenergy emission as observed by the Large Area Telescope (LAT) onboard Fermi (as shown in fig. 1). This sets them apart from GRBs of comparable low-energy fluence, such as GRB 080916C or GRB 090926 [10], which present also a bright high-energy emission. Moreover, for the two GRBs considered here and again in contrast with the other LAT-detected bright GRBs, the long lasting high-energy emission picks up very late, well after the prompt emission has finished. This highenergy component is believed to originate from a different region and due to a different mechanism with respect to the prompt emission. Therefore, we have here the possibility of studying the prompt emission without contamination from the high-energy component.

Since the scenario originating GRBs is still uncertain, as detailed in section 1, we first describe in section 2.1 the spectrum of the two GRBs phenomenologically, without depending on a specific physical picture. Then, in section 2.2, we adopt two specific models (among many possibilities) and, starting from first principles, we fit their physical parameters to the data in order to verify whether they are viable physical pictures for these two GRBs. 

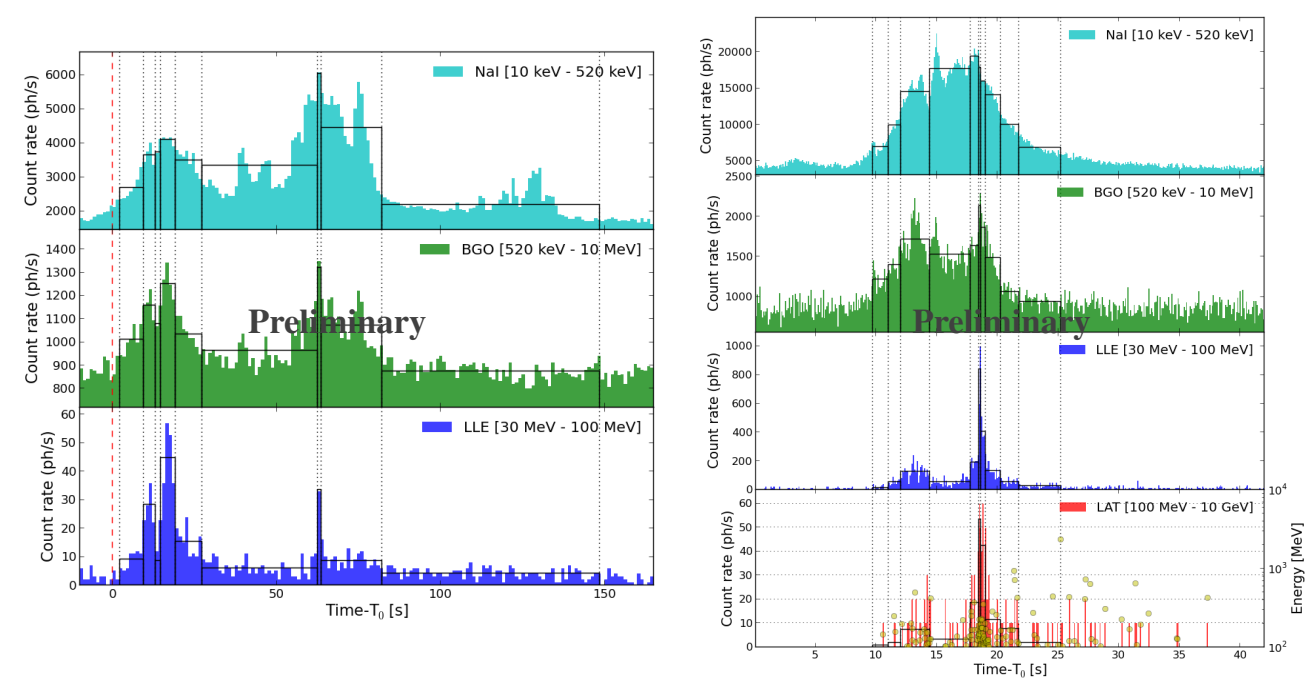

Figure 1: Left: Composite light curve of GRB 100724B showing NaI, BGO and LAT/LLE data. There is no photon spatially and temporally associated with the GRB with energy above $100 \mathrm{MeV}$, thus we do not show LAT standard data. The dashed red vertical lines represent the trigger time, while the other vertical lines correspond to the intervals for our time-resolved spectral analysis. Right: similar plot for GRB 160509A. This time there is a signal in LAT standard data (above $100 \mathrm{MeV}$, shown in the bottom panel). However, most of the photons (yellow dots) have energies below $500 \mathrm{MeV}$.

\subsection{Phenomenological modeling}

The time-integrated spectrum of both GRBs is historically well modeled by a Band spectrum [11]:

$$
f_{\text {Band }}(E)=K\left\{\begin{array}{ll}
E^{\alpha} \exp \left(\frac{-E}{E_{0}}\right) & E<(\alpha-\beta) E_{0} \\
{\left[(\alpha-\beta) E_{0}\right]^{\alpha-\beta} \exp (\beta-\alpha) E^{\beta}} & E \geq(\alpha-\beta) E_{0}
\end{array},\right.
$$

This spectrum models well the low-energy part of both GRBs, but significantly overestimate the emission in the $>10 \mathrm{MeV}$ energy range. Therefore, we consider a model with $f_{\text {Band }}$ multiplied by an exponential cutoff:

$$
f_{B H e c}(E)=f_{\text {Band }} \exp \left(-\frac{E}{E_{c}}\right)
$$

The significance of the cutoff with respect to the Band model alone is much larger than $5 \sigma$ for both GRBs. We have tried also many other phenomenological models, but the $f_{B H e c}$ spectrum provides consistently a better description of the data with the minimum number of parameters. We have also performed a time-resolved spectral analysis in the time intervals indicated in fig. 1. The $f_{B H e c}$ model describes successfully the spectrum in all time intervals with the least number of parameters with respect to the other phenomenological functions. The significance of the cutoff in the time-resolved analysis is larger than $4 \sigma$ in all intervals with good statistic. We also performed a goodness of fit evaluation using Monte Carlo simulations, and we have found that the probability of obtaining a fit equal or worse than what we observe in the data if $f_{B H e c}$ is the true model is $>0.1$ 

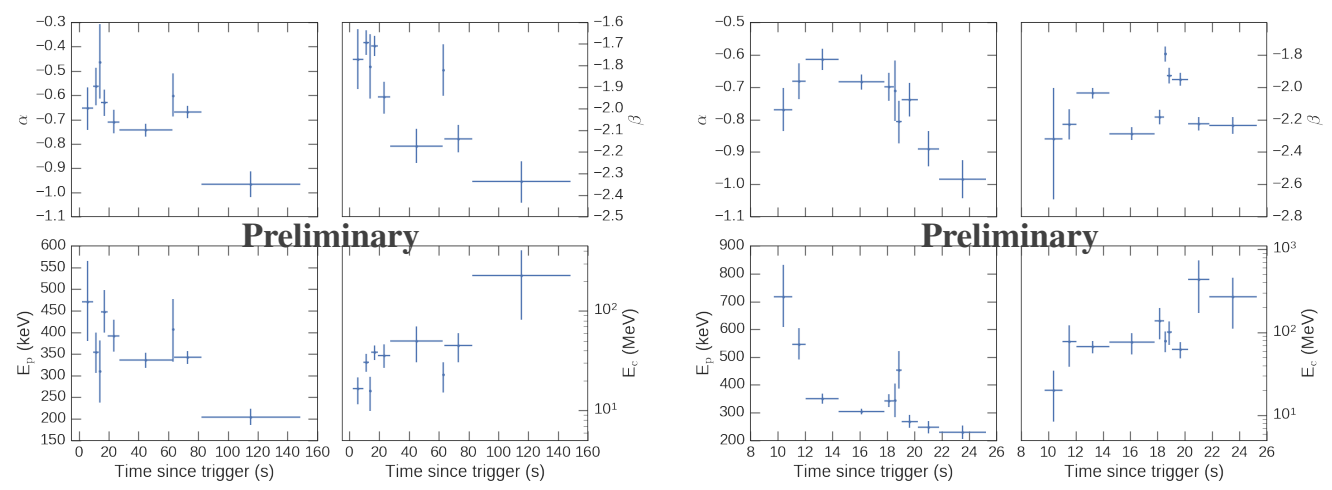

Figure 2: Temporal evolution of the parameters of the $f_{B H e c}$ model (eq. 2.2) for GRB 100724B (left) and GRB 160509A (right).

in all time intervals. We therefore conclude that the $f_{B H e c}$ model is a satisfactory description of the data in all intervals. We show in fig. 2 the best fit parameters for the time-resolved analysis, and in the upper panels in fig. 3 the $v F_{v}$ spectra for the time-resolved analysis. The two GRBs show a similar behavior: there is a general hard-to-soft evolution in both GRBs (left panels), and the cutoff energy $E_{c}$ increases slightly with time (bottom right panels) suggesting a source that is becoming less compact.

\subsection{Physical modeling}

In both the internal-shock and the photospheric frameworks a cutoff such as the one observed in GRB 100724B and GRB 160509A can be explained through opacity effects. In this section we consider, among many possibilities: i) the semi-phenomenological internal-shock model of [12] featuring a detailed modeling of pair production opacity which accounts for the opacity build up during a pulse in the light curve, and ii) the photospheric model of [7], where the cutoff is originated by delayed pair breakdown in a highly-magnetized jet. These models provide a natural explanation for the spectral cutoff, and we have readily available numerical codes which provide the spectra foreseen by the two scenarios as a function of physical parameters. In particular, we obtain direct estimates of the bulk Lorentz factor $\Gamma$ from both models. The internal-shock model also provide a direct estimate of the emission radius $R$ and of the relative size of the emission region. Details about the models as well as the fitting procedure can be found in [9].

We show in the middle and bottom panels in fig. 3 the $v F_{v}$ spectra for the best fit models for each time interval for both GRBs. The photospheric model (middle panels) looks more similar to the phenomenological model (upper panels) than the internal shock model (lower panels). Despite their different shape, however, we find that the quality of the fit obtained with the two physical models is very similar to the one obtained with the phenomenological model. Indeed, despite their differences in the $v F_{v}$ spectrum, these models result in remarkably similar counts spectra due to the energy dispersion effects in both the LAT and the GBM. An example for one interval is shown in fig.4.

For both models we obtain values for the physical parameters self consistent with the assumptions of the two models. For example, for GRB 160509A and the internal shock scenario we obtain 


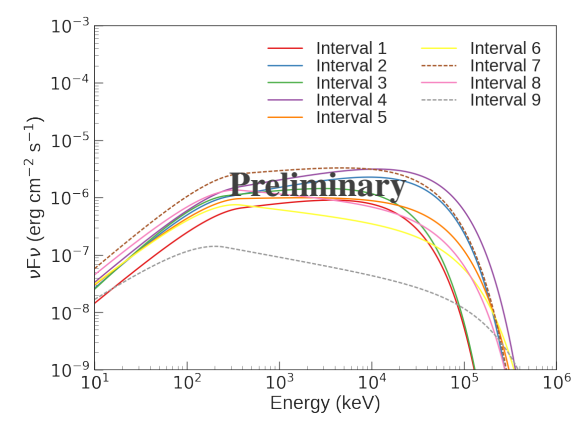

(GRB 100724B, phenomenological $f_{B H e c}$ model)

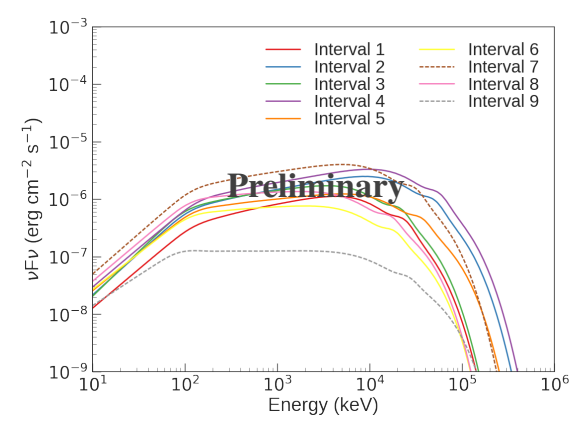

(GRB 100724B, photospheric model)

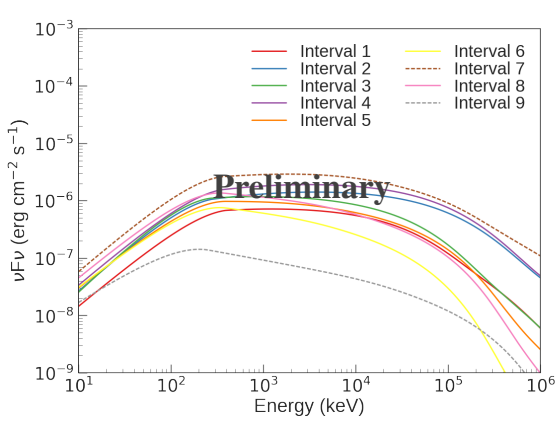

(GRB 100724B, internal-shock model)

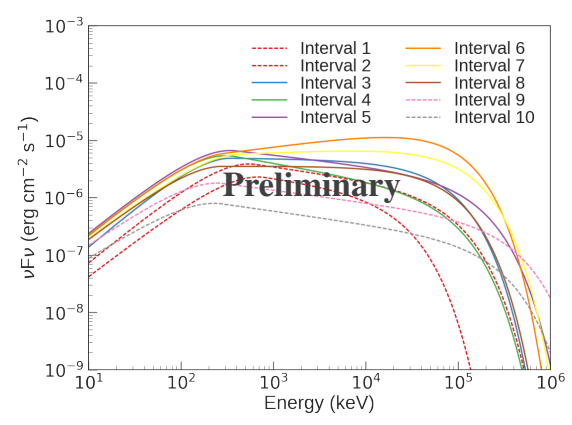

(GRB 160509A, phenomenological $f_{B H e c}$ model)

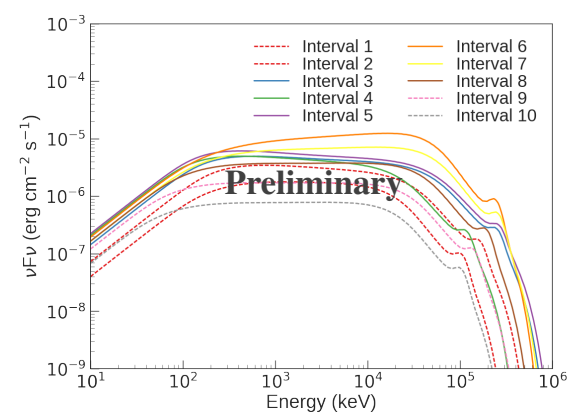

(GRB 160509A, photospheric model)

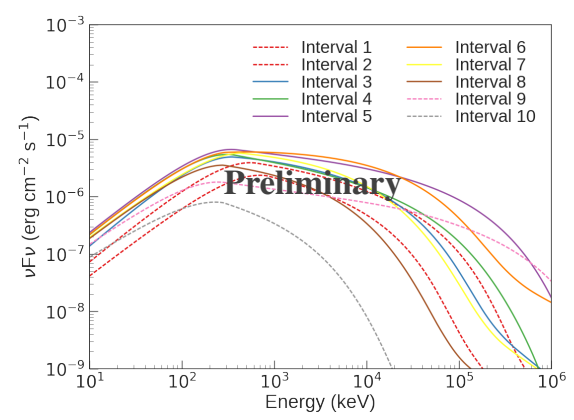

(GRB 160509A, internal-shock model)

Figure 3: Best fit $v F_{v}$ spectra for GRB 100724B (left) and for GRB 160509A (right), for the three models considered in this work. The dashed lines mark intervals where the improvement given by the addition of the cutoff is lower than $3 \sigma$.

an emission radius between $\sim 10^{13}$ and $\sim 10^{14} \mathrm{~cm}$, well in agreement with the expectations [13]. We also can measure directly the bulk Lorentz factor of the jet as a function of time. We report in fig. 4 the results for the two physical models, and for the analytic model of [14] which is normally used to estimate the Lorentz factor for GRBs. The latter case corresponds to a simple one-zone model in which the radiation in the outflow's frame is uniform, isotropic and time-independent. This model consistently yields a higher Lorentz factor than the internal shock model with pair production opacity that we have considered, where the radiation field starts from zero at the emission onset and is calculated self-consistently as a function of time, space and direction. The photo- 

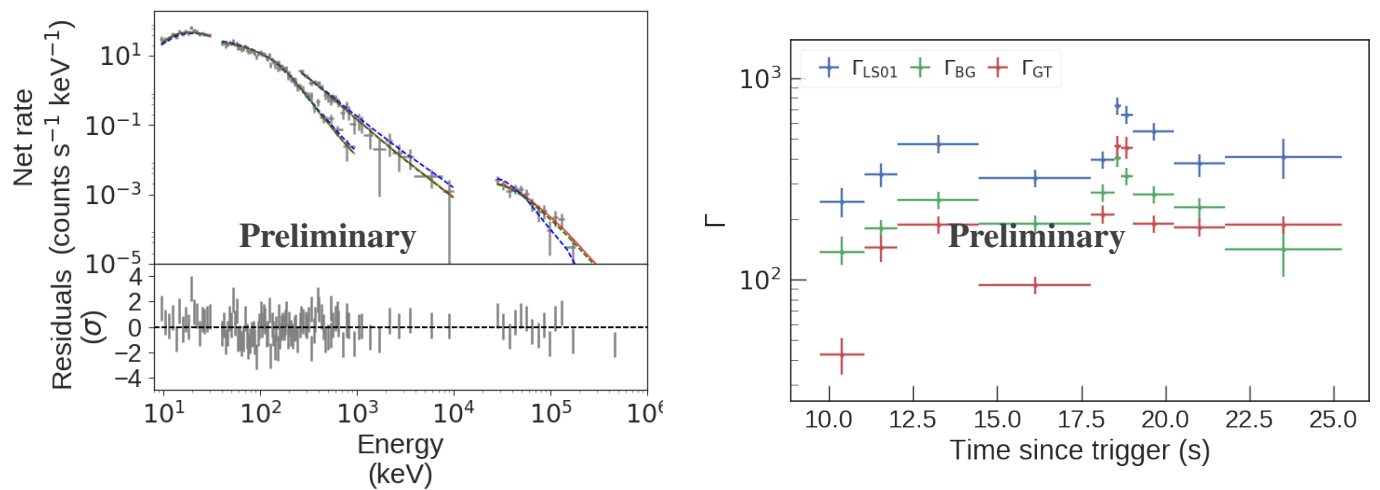

Figure 4: (Left) Count spectra for interval 6 of GRB 160509A. The red line corresponds to the phenomenological model $f_{B H e c}$, while the blue and the green dashed lines correspond respectively to the internal shock and the photospheric models. (Right) Comparison of the bulk-LFs obtained from three different models for GRB 160509A. The three models are the semi-analytic internal-shock model with pair production opacity of [12] (BG), the photospheric model of [7] (GT), and (LS01) analytic model of Lithwick \& Sari (2001). For all time intervals, LS01 consistently yields the highest $\Gamma$.

spheric model gives a even lower estimate for the Lorentz factor. The GRBs reported here are the two best examples of high-energy cutoffs in LAT GRBs. The others LAT GRBs with comparable low-energy fluence have either higher Lorenz factors, or higher lower limits on the Lorentz factor [10]. For example the bright GRB 080916C has $\Gamma>600$ throughout the burst, while GRB 090902 has $\Gamma>1000$.

\section{Conclusions}

GRB 100724B and GRB 160509A are two bright LAT-detected GRBs showing a clear cutoff in the sub-GeV energy range during the prompt emission. By using a phenomenological model, independent of physical assumptions, we measure a cutoff in the range 20-60 MeV and 80-150 $\mathrm{MeV}$ respectively. This cutoff can be interpreted as a due to pair production opacity at the source. In particular, the semi-phenomenological model of [12], which features a detailed and self-consistent computation of $\gamma \gamma$ opacity in a highly variable source, describes the data well and yields results in agreement with the expectations of the internal shock model. The photospheric model that we have considered, featuring opacity deriving from the delayed pair breakdown in a highly-magnetized jet and described in details in [7], can also describe very well the data. We cannot therefore rule out either explanation. The estimate of the bulk Lorentz factors derived with these models are a factor of a few to several smaller than the measurements or the lower limits derived for bright LAT-detected GRBs. Because of opacity to intrinsic pair production, slower GRBs tend to be fainter in the LAT energy range while they can be just as bright in the GBM energy range, and are therefore more difficult to detect for the LAT. This may produce a selection bias against deriving lower Lorentz factors from the detection of high-energy cutoffs. 


\section{References}

[1] P. Kumar and B. Zhang, The physics of gamma-ray bursts and relativistic jets, Phys. Rep. 561 (Feb., 2015) 1-109, [1410.0679].

[2] S. Kobayashi and R. Sari, Ultraefficient Internal Shocks, ApJ 551 (Apr., 2001) 934-939, [astro-ph/0101006].

[3] R. D. Preece, M. S. Briggs, T. W. Giblin, R. S. Mallozzi, G. N. Pendleton, W. S. Paciesas et al., On the Consistency of Gamma-Ray Burst Spectral Indices with the Synchrotron Shock Model, ApJ 581 (Dec., 2002) 1248-1255.

[4] J. M. Burgess, F. Ryde and H.-F. Yu, Taking the band function too far: a tale of two $\alpha$ 's, MNRAS 451 (Aug., 2015) 1511-1521, [1410 . 7647].

[5] M. Axelsson and L. Borgonovo, The width of gamma-ray burst spectra, MNRAS 447 (Mar., 2015) 3150-3154, [1412.5692].

[6] D. Giannios, The peak energy of dissipative gamma-ray burst photospheres, MNRAS 422 (June, 2012) 3092-3098, [1111.4258].

[7] R. Gill and C. Thompson, Non-thermal Gamma-Ray Emission from Delayed Pair Breakdown in a Magnetized and Photon-rich Outflow, ApJ 796 (Dec., 2014) 81, [1406.4774].

[8] P. Veres, B.-B. Zhang and P. Mészáros, Magnetically and Baryonically Dominated Photospheric Gamma-Ray Burst Model Fits to Fermi-LAT Observations, ApJ 764 (Feb., 2013) 94, [1210.7811].

[9] G. Vianello, R. Gill, J. Granot, N. Omodei, J. Cohen-Tanugi and F. Longo, The Bright and the Slow - GRBs 100724B and 160509A with high-energy cutoffs at $\lesssim 100 \mathrm{MeV}, \mathrm{ArXiv}$ e-prints (June, 2017), [1706.01481].

[10] M. Ackermann, M. Ajello, K. Asano, M. Axelsson, L. Baldini, J. Ballet et al., The First Fermi-LAT Gamma-Ray Burst Catalog, ApJS 209 (Nov., 2013) 11, [1303.2908].

[11] D. Band, J. Matteson, L. Ford, B. Schaefer, D. Palmer, B. Teegarden et al., BATSE observations of gamma-ray burst spectra. I - Spectral diversity, ApJ 413 (Aug., 1993) 281-292.

[12] J. Granot, J. Cohen-Tanugi and E. do Couto e Silva, Opacity Buildup in Impulsive Relativistic Sources, ApJ 677 (Apr., 2008) 92-126, [0 708 . 4228].

[13] M. J. Rees and P. Meszaros, Unsteady outflow models for cosmological gamma-ray bursts, ApJL 430 (Aug., 1994) L93-L96, [astro-ph/ 9404038 ].

[14] Y. Lithwick and R. Sari, Lower Limits on Lorentz Factors in Gamma-Ray Bursts, ApJ 555 (July, 2001) 540-545, [astro-ph/0011508]. 